\title{
Revisión sistemática \\ Eficacia de las intervenciones farmacológicas para el tratamiento de la COVID-19
}

\author{
DOI 10.5377/alerta.v4i2.10904 \\ Oscar Caballero-Calderón ${ }^{1 *}$, Ana García-Sura ${ }^{2}$ \\ 1. Unidad Comunitaria de Salud Familiar, Concepción de Ataco, Ahuachapán, El Salvador. \\ 2. Consultora independiente, San Salvador, El Salvador \\ ${ }^{*}$ Correspondencia \\ $\nabla$ oscarcaballero2@gmail.com
}

(1) 0000-0001-5105-8964

2. (1) 0000-0002-8828-9843

\begin{abstract}
Resumen
Introducción. A pesar de la elevada morbimortalidad por coronavirus 19 aún no existe un tratamiento eficaz para abordarla. Objetivo. Establecer la eficacia de las intervenciones farmacológicas en el tratamiento de adultos con diagnóstico de enfermedad por coronavirus en cualquier fase. Metodología. Mediante una revisión exploratoria, se examinaron publicaciones hasta el 21 de enero de 2021 por una búsqueda en MEDLINE, Cochrane, me-dRxiv, New England Journal vía PubMed. Se analizaron fármacos reductores de la actividad viral, corticoides, terapia relacionada al sistema inmune para evaluar los desenlaces de supervivencia, ventilación mecánica, estancia hospitalaria y seguridad tras su aplicación a pacientes en fase leve, moderada y/o grave de la enfermedad. Se priorizaron ensayos clínicos controlados y aleatorizados, cuyo riesgo de sesgo se determinó con Newcastle-Ottawa y A measurement Tool to Assess Systematic Reviews 2. Resultados. Se comprobó que el Interferon-a2b disminuye la duración de eliminación del virus y los marcadores inflamatorios. En la terapia autoinmune, el tocilizumab mostró leve eficacia al administrarlo de forma única, sin embargo, combinado con dexametasona potencia su efecto. Conclusión. A un año de la pandemia por coronavirus 19 no hay evidencia concluyente sobre su terapia. Se ha comprobado cierta eficacia del Interferon-a2b inhalado, así como del tocilizumab y la dexametasona en administración única o combinada.

Palabras clave

COVID-19, tratamiento, antivirales, corticoesteroides.
\end{abstract}

f ACCESO ABIERTO

Efficacy of pharmacological interventions for the treatment of COVID-19

\section{Citación recomendada:} Caballero Calderón O, García Sura A. Eficacia de las intervenciones farmacológicas en el tratamiento de la COVID-19. Alerta.2021;4(2) 81-87. DOI: 10.5377/alerta. v4i2.10904

Recibido:

22 de febrero de 2021

\section{Aceptado:}

13 de mayo 2021

\section{Publicado:}

21 de mayo 2021

\section{Contribución de autoría:} AGS: Planificación y elaboración del estudio, elaboración borrador manuscrito. OCC: recolección y análisis de información, correcciones del manuscrito

Conflicto de intereses: Autores niegan tener algún conflicto de interés

\section{Introducción}

Actualmente, las intervenciones farmacológicas constituyen uno de los pilares fundamentales en el tratamiento de la enfermedad por coronavirus (COVID-19), sin embargo, hasta el 21 de enero de 2021 la Organización Mundial de la Salud (OMS) reportó 95612831 casos de la COVID-19 a nivel global, de los cuales 610403 fueron nuevos casos y 2066176 defunciones'. Esto refleja, que los medicamentos disponibles para reducir la morbilidad y mortalidad por la COVID-19, no son eficaces y se necesita establecer un abordaje farmacológico específico para tratarla.

En los primeros casos, el tratamiento fue seleccionado con base a las experiencias obtenidas en pacientes con Síndrome Respiratorio Agudo Severo (SARS por sus siglas en inglés), Síndrome Respiratorio de Oriente Medio (MERS por sus siglas en inglés) y procedentes de estudios preclínicos (in vitro), aunque, la evidencia señaló que no son eficaces ni seguros para tratar la COVID-19². 
Hoy en día, las intervenciones son elegidas en función de las fases de la enfermedad a partir de los fármacos que evitan que el virus entre a las células que evitan su replicación y los que actúan en la fase inflamatoria que reducen el daño a órganos. Estos se dividen en cuatro familias de fármacos: antivirales, corticoesteroides, anticoagulantes y la terapia relacionada al sistema inmune ${ }^{3}$, cuyo alcance y seguridad siguen siendo estudiados.

Por este motivo, se realizó una revisión tomando en cuenta la literatura sobre el tratamiento de pacientes adultos en diferentes etapas de la COVID-19, evaluando cuál es la eficacia de cada una de ellas mediante los desenlaces de supervivencia, duración de la hospitalización, reducción de la necesidad de respiración mecánica y la seguridad de las mismas, a fin de identificar posibles intervenciones terapéuticas que disminuyan complicaciones severas o la muerte de los pacientes.

En este contexto, se examinaron diferentes bases de datos con el objetivo de establecer la eficacia de las intervenciones farmacológicas en el tratamiento de pacientes adultos con diagnóstico de la COVID-19 en cualquier fase de la enfermedad.

\section{Metodología}

\section{Estrategia de búsqueda}

("severe acute respiratory syndrome coronavirus 2" [Supplementary Concept]) OR ("COVID-19 drug treatment" [Supplementary Concept]) AND "Chloroquine" [Mesh] ("Hydroxychloroquine" [Mesh]) AND "COVID-19" [Supplementary Concept] AND ("Favipiravir" [Mesh] AND "Lopinavir/ritonavir" [Mesh] AND "Remdesivir" [Mesh] AND "Interpheron - a 2b" [Mesh] AND"Ivermectin" [Mesh] AND "corticosteroids" [Mesh] OR "dexamethasone" [Supplementary Concept]) AND "convalescent plasma" AND "vitamin C" OR "ascorbic acid" [Supplementary Concept] AND "tozilizumab")

\section{Criterios de elegibilidad}

\section{Criterios de inclusión}

- Estudios desarrollados en pacientes adultos con diagnóstico de COVID-19 durante cualquier etapa de la enfermedad.

- Artículos publicados del 1 de enero 2020 al 21 de enero de 2021.

- Versiones definitivas con texto completo sobre intervenciones farmacológicas en la COVID-19 sin restricción de idioma.

- Ensayos clínicos aleatorios y no aleatorios, revisiones sistemáticas.

\section{Criterios exclusión}

- Investigaciones en personas con Síndrome respiratorio de Oriente Medio (MERS), Síndrome respiratorio agudo severo (SARS).

- Revisiones narrativas, estudios preclínicos (estudios in vitro o en modelos animales), artículos de opinión, consenso de expertos o guías clínicas.

- Estudios sobre tratamiento adyuvante para manejo de complicaciones de la COVID-19 o tratamiento de soporte.

\section{Búsqueda y selección de la infor- mación}

Se realizó una búsqueda de forma digital en las bases de datos Medline, BVS, JAMA, Science Direct, Cochrane, New England Journal vía Pubmed mediante una estrategia que incluyó términos libres y descriptores de lenguaje controlado que se limitó a publicaciones de enero de 2020 al 21 de enero de 2021, sin restricción de idioma. También, se ejecutó de forma manual en las referencias de la bibliografía seleccionada, a fin de rescatar otras publicaciones potencialmente incluibles.

Se identificaron 2552 publicaciones en las fuentes consultadas, de estas, se eliminaron 2289 tras la revisión. Las 263 publicaciones restantes fueron examinadas de texto completo (Figura 1). Se incluyeron 25 que cumplieron criterios de inclusión.

La evidencia fue seleccionada por dos autores que consideraron una fase de lectura de títulos y resúmenes obtenidos en la búsqueda, seguida de una fase de lectura de texto completo de los estudios potencialmente relevantes mediante consenso.

\section{Evaluación de riesgo de sesgo}

Se estimó mediante herramientas validadas y estandarizadas según el diseño del estudio:

- Ameasurement Tool to Assess Systematic Reviews (AMSTAR-2) para las revisiones sistemáticas (alta, moderada, baja y críticamente baja)

- Riesgo de sesgo 2 (Rob 2) en ensayos clínicos

\section{Variables del estudio}

- Eficacia: supervivencia, estancia intrahospitalaria, reducción de la necesidad de ventilación mecánica.

- Seguridad (efectos adversos graves)

- Intervenciones farmacológicas: antivirales, anticoagulantes, corticoesteroides, terapia relacionada con el sistema inmune. 
Figura 1. Flujo de selección de los estudios según la guía PRISMA 2009³.

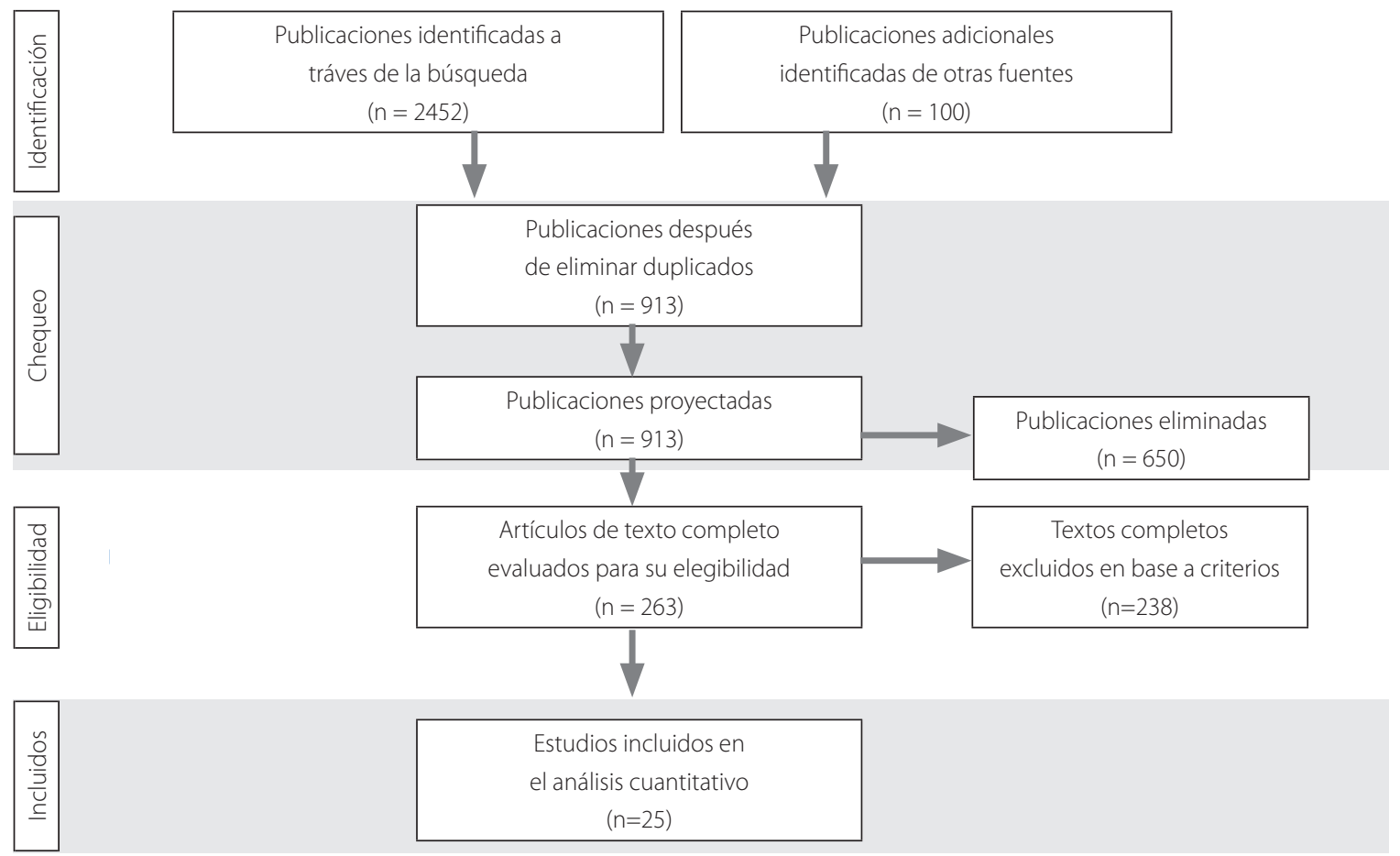

\section{Extracción de datos}

Para extraer la información se utilizó la herramienta Covidence ${ }^{4}$, la cual se depositó en dos matrices elaboradas por los autores en el programa Microsoft Excel 2010; una matriz bibliográfica, que recogió la información del autor, fecha publicación, país de realización, tipo de estudio y otra analítica que concentró las variables en estudio, número de participantes, características de la población, intervención farmacológica, desenlaces principales (eficacia), efectos adversos.

\section{Resultados}

Se revisaron 25 estudios sobre la eficacia de intervenciones farmacológicas para tratar COVID-19 en los que se incluyeron: 15 ensayos clínicos aleatorizados, ocho no aleatorizados, dos revisiones sistemáticas y tres estudios observacionales siguiendo las pautas de Preferred Reporting Items for Systematic Reviews and Meta-Analyses (PRISMA)

\section{Intervenciones farmacológicas}

\section{Fármacos reductores de la carga viral Hidroxicloroquina (HCQ)}

Una revisión sistemática que incluyó 12 ensayos clínicos e incorporó 8569 personas adultas, reportó que no encontraron diferencia significativa en la reducción de contagios comprobados con prueba PCR para SARS-CoV-2 a los 14 días de iniciado el tratamiento (RR 1,00, $95 \% \mathrm{Cl} 0,91-1,10) \mathrm{ni}$ en la disminución de la progresión a ventila- ción mecánica en los pacientes tratados con HCQ (RR 1,11, 95 \% Cl 0,91 - 1,37). En cambio, el riesgo de efectos adversos fue tres veces superior para la HCQ comparado con el tratamiento estándar ${ }^{5}$.

Un ensayo clínico aleatorizado en el que se evaluaron 30 pacientes en fase moderada de COVID-19, reveló que no se encontraron diferencias en el aclaramiento viral nasofaríngeo en el día siete $(86,7 \%$ en pacientes tratados con HCQ y 93,3\% en el grupo de control, $P>0,05)$. Así mismo, no hubo diferencias en la conservación del ARN del virus (mediana 4 días). Ni en los efectos adversos (4 casos del grupo de HCQ y 3 casos del grupo de control tuvieron diarrea transitoria y función hepática anormal) ${ }^{6}$.

Otro ensayo clínico que incorporó 81 pacientes en fase severa reveló una mortalidad del $39 \%$ (16/41) en el grupo tratado con altas dosis de HCQ (log rank-2,183; P: 0,03) y alteraciones electrocardiográficas, con elevación del QT en 11/73 pacientes (18,9\%). Las limitaciones del estudio incluyen la falta de un grupo control placebo, el pequeño tamaño de la muestra, la ausencia de criterios de exclusión basados en el intervalo QT al inicio del estudio?.

\section{Faviripiravir (FPV)}

Un ensayo clínico que incorporó 91 pacientes reveló que el tiempo medio de aclaramiento viral para los pacientes tratados con FPV fue de 4 días (IQR: 2,5 - 9), ( $<<0,001)$. Asimismo, que no se encontró diferencia significativa en las tasas de mejora en los días 4 
y $8(P>0,05)$ hasta el día 14 después del tratamiento $(91,4 \% \text { del FPV, 32/35; } \mathrm{P}=0,004)^{8}$.

\section{Lopinavir/ Ritonavir (LPV/RTV)}

Un ensayo clínico reportó que en 199 casos hospitalizados de neumonía no existió beneficio clínico importante al tratar los pacientes con Lopinavir/ Ritonavir que con la forma estándar (oxígeno suplementario, ventilación invasiva o no invasiva, antibióticos, soporte vasopresor, terapia de reemplazo renal y oxigenación por membrana extracorpórea) ${ }^{9}$.

El ensayo SOLIDARITY de la OMS que involucró 1411 pacientes hospitalizados reportó que las tasas de mortalidad de lopinavir fue de $R R=1,00$ (IC 95\% de 0,79-1,25; $\mathrm{P}$ $=0,97 ; 148 / 1399$ frente a 146/1372) ${ }^{10}$.

El ensayo RECOVERY por su parte, reveló que no encontró diferencia significativa entre el tiempo de alta del hospital (mediana de 11 días [IQR 5 a >28]) o la proporción de pacientes dados de alta del hospital vivos dentro de 28 días (relación de velocidad $0,98 ;$ IC $0,91-1.05 ; p=0,53)^{11}$.

Asimismo, que no se comprobó la diferencia entre los pacientes que en un inicio no estaban en ventilación mecánica invasiva con la proporción de aquellos con ventilación mecánica invasiva o muerte (relación de riesgo 1,09, IC $95 \% 0,99-1,20 ; p=0,092)^{12}$.

\section{Remdesivir}

Un ensayo doble ciego aleatorizado y controlado en 1062 pacientes hospitalizados con afectación del tracto respiratorio bajo en la fase crítica de la enfermedad, reportó que la administración del Remdesivir redujo el tiempo de recuperación ${ }^{13}$.

Además, que, en la fase moderada de la enfermedad, reportó que no hay diferencia en el estado del paciente al aplicar tratamiento estándar para la COVID-19 o el Remdesivir ${ }^{12}$

En 53 pacientes con COVID-19 tratados, de los cuales 30 estaban siendo ventilados y 4 tratados con oxigenación por membrana extracorpórea al inicio del tratamiento un ensayo clínico reveló que después de una mediana de 18 días, 25/53 los pacientes (47\%) fueron dados de alta del hospital y 7 (13\%) habían muerto. La mortalidad fue del $5 \%$ entre los pacientes que no fueron ventilados. La probabilidad general de mejora en 18 días fue del $68 \%$ (IC $95 \%, 40 \%$ a $80 \%) .32$ pacientes (60 \%) tuvieron al menos un evento adverso de los que en 12 (23\%) fueron graves ${ }^{13}$.

Otro ensayo clínico aleatorizado en 2750 pacientes hospitalizados (SOLIDARITY) reportó que tras administrar Remdesivir no se reportó la reducción de la mortalidad (301/2743; 10,97\%), [hazard ratio de 0,95
(IC $95 \% ; 0,81$ - 1,11)]. Tampoco la reducción de la progresión al uso de ventilación mecánica. (295/2489 - 11,9 \% vs 284/2475 $11,5 \%)^{10}$.

\section{Interferón - a 2b. (IFN-a2b)}

En casos moderados de la COVID-19, un estudio exploratorio no controlado reveló que el Interferón-a2b inhalado acorta la duración de eliminación del virus y reduce los marcadores de inflamación aguda como la proteína $C$ reactiva (PCR) y la interleucina-6 (IL-6) ${ }^{14}$. Las limitaciones de la investigación incluyen que es una cohorte pequeña, y no aleatoria ${ }^{14}$.

\section{Ivermectina}

Un ensayo clínico aleatorizado que evaluó 476 pacientes reportó que no comprobó diferencia significativa en la resolución de los síntomas, mediana de 10 días (IQR 9 - 13) al administrar ivermectina; $\mathrm{p}=0,53$ mediante la prueba de rango logarítmico. Así también, que el evento adverso más común en 104 pacientes (52\%) que recibieron ivermectina fue el dolor de cabeza y en 111 (56\%) y el más grave fue el fallo multiorgánico, que se produjo en 4 pacientes ( 2 en cada grupo) ${ }^{15}$.

Otro ensayo clínico aleatorizado que incorporó 24 pacientes reveló que no hubo diferencia en la proporción de pacientes con PCR positivo en el día 7 después del tratamiento (RR: 0,92, $95 \% \mathrm{Cl}: 0,77-10,09, \mathrm{p}=1)^{16}$.

\section{Corticoesteroides}

El ensayo "Randomised Evaluation of COViD-19 thERapY" (RECOVERY) que incluyo 2104 pacientes hospitalizados reportó que la dexametasona, redujo la tasa de mortalidad en 1 de cada 8 pacientes con ventilación mecánica que presentaron duración de síntomas mayor a 7 días $(0,7 ; \mathrm{IC}$ del $95 \%$ 0,5 a 0,$9 ; p=0,0003$ ) y en 1 de cada 25 de los casos que requirieron oxigenoterapia $(0,8 ; \mathrm{IC}$ del $95 \% 0,7$ a $1 ; p=0,002)^{16}$ disminuyó la mortalidad. No se reportaron beneficios al administrar dexametasona en casos que no requirieron oxigeno (fases leves, moderada de la enfermedad) ${ }^{17}$.

\section{Terapias relacionadas al sistema inmune Plasma sérico convaleciente}

Dos estudios, que incluyeron 10 y 6 casos graves de COVID-19, reportaron que el plasma convaleciente provocó la disminución o eliminación de los síntomas en 3 días, el desaparecimiento de la viremia y diversos grados de absorción de lesiones pulmonares en el examen radiológico en 7 días, así como un incremento en los títulos séricos de $\lg G$ e $\lg \mathrm{M}^{18,-19}$.

Un estudio multicéntrico también reportó que el tratamiento con plasma convale- 
ciente mejoró la supervivencia de los pacientes, redujo el período de hospitalización y de la necesidad de intubación ${ }^{20}$.

El ensayo clínico PLACID que incluyó casos hospitalizados durante la fase moderada, registro que la administración de plasma convaleciente no disminuyó la mortalidad ni la progresión a enfermedad grave, aunque mejoró la dificultad para respirar, la fatiga y condujo a una mayor conversión negativa del ARN del SARS-CoV-221.

\section{Vitamina C}

Un estudio realizado en pacientes hospitalizados con Síndrome Respiratorio Agudo (SDRA), a quienes se administró una dosis 100 veces más alta que la máxima alcanzada mediante la administración de suplementos orales, reveló que en el 16,5\% de ellos se redujo la mortalidad y en el 2,5\% la necesidad de asistencia respiratoria mecánica. También, reportó que redujo en 3,2 días la estancia en cuidados intensivos y 6,7 días de hospitalización ${ }^{21}$. Se tiene como limitación, la baja potencia del estudio ${ }^{22}$.

\section{Tozilizumab (TCZ)}

Una investigación llevada a cabo en 21 pacientes con neumonía grave reportó que el $\mathrm{TC} Z$ reduce la necesidad de ventilación mecánica ${ }^{23}$.

El ensayo Recovery, reportó que en 2022 pacientes con niveles bajos de oxígeno e inflamación que recibieron tocilizumab redujo el número de muertes en un $29 \%$ ( $n=596$ ). También, que incrementó la probabilidad del alta a los 28 días en el $54 \%(n=1091)$ de los pacientes y disminuyó el progreso a ventilación mecánica invasiva o muerte del $38 \%$ al $33 \% 24$.

Este ensayo, señala que al administrar dexametasona en combinación con tozilizumab reduce la mortalidad en aproximadamente $1 / 3$ de los pacientes que requieren oxígeno simple y en la mitad de los que requieren ventilación mecánica invasiva ${ }^{24}$.

\section{Discusión}

Se identificaron las intervenciones farmacológicas que han mostrado probable eficacia en el tratamiento de la COVID-19 (el Interferón-a2b inhalado para casos moderados $^{14}$. Para etapas graves, el tozilizumab y la dexametasona en administración única o combinados) $)^{23,-24}$.

Se comprobó que los fármacos que reducen la carga viral como el Remdesivir aceleran la recuperación clínica, acortando la duración de la hospitalización, especialmente en pacientes que precisan oxigenoterapia suplementaria ${ }^{12,-13}$, sin embargo, el ensayo
Solidaridad (SOLIDARITY) reportó que no es eficaz, pues no incrementó la supervivencia, ni acortó la estancia hospitalaria, ni redujo la necesidad de ventilación mecánica durante el ensayo ${ }^{8}$. Estos resultados podrían considerarse no concluyentes, al no tener un diseño doble ciego ${ }^{19}$.

Cabe señalar, que algunos estudios reportan que presenta como efectos adversos anemia, lesión renal aguda, disminución de la filtración glomerular, elevación de la AST y/o la $\mathrm{ALT}^{13}$, lo cual implica que no es $100 \%$ seguro, por tanto, si bien su eficacia no es concluyente, deben considerarse las comorbilidades del paciente y las interacciones medicamentosas, así como el beneficio/costo de su uso antes de elegirlo como terapia.

En esta misma familia, la cloroquina y/o la hidroxicloroquina administrados de forma única o combinada con un macrólido (azitromicina, claritromicina), el favipiravir junto a lopinavir/ritonavir ${ }^{31}$; por su baja actividad antiviral en todas las fases de la COVID-19 y su poca seguridad (efectos adversos que ponen en riesgo la vida de los pacientes) se eliminaron de los protocolos de tratamiento ${ }^{5-7}$.

La calidad baja-moderada de la evidencia ${ }^{21-25}$ puede refutar estos resultados, sin embargo, se ha demostrado que los fármacos no son seguros y su reutilización en combinación con inmunomoduladores que posibiliten potenciar su efecto no es viable, ya que el riesgo para el paciente es alto.

Por otra parte, los estudios disponibles de la ivermectina no han comprobado su eficacia en el tratamiento del COVID-19 leve (5-16. $^{15}$

En la terapia relacionada al sistema autoinmune, el tozilizumab ha mostrado cierta eficacia en pacientes con ventilación mecánica, ya que, al administrarlo de forma única en etapas graves, reduce la mortalidad en un $4 \%$, aunque, al combinarlo con dexametasona potencia su actividad antiinflamatoria produciendo reducción de la mortalidad en la mitad de los casos².

La seguridad del tozilizumab podría cuestionarse por la calidad de la evidencia, pero su combinación con la dexametasona constituye hasta la fecha, la única terapia disponible para hacer frente a los efectos inflamatorios por la COVID-19 que han revelado eficacia ${ }^{23,24}$.

Otras intervenciones, como el plasma convaleciente en diferentes estudios han mostrado efectos favorables, principalmente en casos graves de la COVID-19, sin embargo, no es posible determinar su eficacia, ya que, en la mayoría de ensayos clínicos analizados, no pudo ser comprobada. Debido a su bajo costo, ausencia de efectos adversos, en algunos países es la primera opción para tratar casos graves de la COVID-19 ${ }^{18-21}$. 
La vitamina C, ha mostrado efectos favorables al aplicarla en pacientes con Síndrome Respiratorio Agudo29, aunque, estos hallazgos no pueden considerarse como concluyentes, pues el ensayo no tuvo la suficiente potencia ${ }^{22-24}$.

En muchos países, este fármaco es parte del tratamiento de la fase leve de la enfermedad, posiblemente por su efecto antioxidante y sobre el sistema inmune, pero aún no hay evidencia que respalde o refute su uso ${ }^{24}$.

Asimismo, los corticoesteroides han demostrado eficacia para tratar la fase inflamatoria, aunque por los efectos adversos de éstos, se prefiere utilizar la dexametasona, debido a que retiene menor cantidad de agua y sodio ${ }^{17}$.

Al administrarla de forma única, la dexametasona reduce la mortalidad de pacientes en etapas graves de la enfermedad, especialmente en los que requieren oxigenoterapia y/o ventilación mecánica ${ }^{17,24}$.

Estos hallazgos a la fecha son concluyentes, pues en diferentes estudios, por ejemplo, el RECOVERY, el efecto sobre el sistema inmunitario y antiinflamatorio de esta familia ha sido comprobada en esta y otras enfermedades. Como ventaja adicional, esta su disponibilidad ${ }^{24}$.

Muchos de los estudios incluidos presentan sesgos de selección y de desgaste por la falta de aleatorización y las pérdidas, lo que sumado a las nuevas mutaciones del SARSCoV-2, estudios de nuevos fármacos por finalizar, provoca que a un año de la pandemia no se cuente con intervenciones farmacológicas específicas para tratar la COVID-19.

Por lo que es necesario realizar estudios en pacientes hospitalizados en los que se hallan detectado mutaciones del virus a fin de comprobar si las intervenciones farmacológicas utilizadas aún son viables.

\section{Conclusiones}

A la fecha, los fármacos disponibles que han mostrado eficacia para tratar la COVID-19, incluyen al Interferón-a2b inhalado para fases moderadas, tocilizumab y la dexametasona administrados de forma única o combinados en etapas graves. La evidencia no es concluyente, lo que hace necesario realizar estudios que incorporen las nuevas cepas del virus y completar los estudios de fármacos en desarrollo.

\section{Agradecimiento}

A las doctoras Ana María Barrientos Llovet y Milena Marquina de Reyes por sus valiosos comentarios en la forma del artículo.

\section{Financiamiento}

El estudio fue realizado con fondos propios de los investigadores.

\section{Referencias bibliográficas}

1. Organización Panamericana de la Salud / Organización Mundial de la Salud. Actualización epidemiológica: Enfermedad por Coronavirus (COVID-19). Washington, D.C. OPS/OMS. 14 de abril de 2021. 25 p. Disponible en: https://www.paho.org/es/ file/85719/download?token=EW9Y6u5l

2. Kupferschmidt K, Cohen J. Race to find COVID-19 treatments accelerates. Science. 2020;367(6485):1412-1413. DOl:10.1126/ science.367.6485.1412Moher D, Liberati A, Tetzlaff J, Altman DG, The PRISMA Group. Preferred reporting items for systematic reviews and meta-analyses: The PRISMA Statement. PLoS Med. 2009;6(7):e1000097. DOI: 10.1371/journal.pmed.1000097

3. Singh B, Ryan H, Kredo T, Chaplin M, Fletcher T. Chloroquine or hydroxychloroquine for prevention and treatment of COVID-19 Cochrane Infectious Diseases Group, editor. Cochrane Database Syst. Rev. 2021 Feb 12. DOI:10.1002/14651858.CD013587.pub2

4. Covidence. Better systematic review management. Covidence. 2021. Fecha de consulta: 2020. Disponible en: https://www. covidence.org/

5. Chen J, Liu D, Liu L, Liu P, Xu Q, Xia L, et al. [A pilot study of hydroxychloroquine in treatment of patients with moderate COVID-19]. Zhejiang Da Xue Xue Bao Yi Xue Ban. 2020;49(2):215-219. DOI: 10.3785 / j.issn.1008-9292.2020.03.03

6. Chen Z, Hu J, Zhang Z, Jiang S, Han S, Yan D, et al. Efficacy of hydroxychloroquine in patients with COVID-19: results of a randomized clinical trial (Preprint). Epidemiology; 2020. DOI: $10.1101 / 2020.03 .22 .20040758$

7. Borba MGS, Val FFA, Sampaio VS, Alexandre MAA, Melo GC, Brito M, et al. Effect of High vs Low Doses of Chloroquine diphosphate as adjunctive therapy for patients hospitalized with Severe Acute Respiratory Syndrome Coronavirus 2 (SARSCoV-2) infection: a randomized clinical trial. JAMA Netw. Open. 2020;3(4):e208857. DOI: 10.1001/jamanetworkopen.2020.8857

8. Cai Q, Yang M, Liu D, Chen J, Shu D, Xia J, et al. Experimental Treatment with Favipiravir for COVID-19: An Open-Label Control Study. Engineering. 2020;6(10):1192-1198. DOI: $10.1016 /$ j.eng.2020.03.007

9. WHO Solidarity Trial Consortium. Repurposed Antiviral Drugs for Covid-19- 
Interim WHO solidarity trial results. N. Engl. J. Med. 2021;384(6):497-511. DOI:10.1056/ NEJMoa2023184

10. Horby PW, Mafham M, Bell JL, Linsell L, Staplin N, Emberson J, et al. Lopinavirritonavir in patients admitted to hospital with COVID-19 (RECOVERY): a randomised, controlled, open-label, platform trial. The Lancet. 2020;396(10259):1345-1352. DOI:10.1016/S0140-6736(20)32013-4

11. Beigel JH, Tomashek KM, Dodd LE, Mehta AK, Zingman BS, Kalil AC, et al. Remdesivir for the treatment of Covid-19 — Final Report. N. Engl. J. Med. 2020;383(19):18131826. DOI:10.1056/NEJMoa2007764

12. Spinner CD, Gottlieb RL, Criner GJ, Arribas López JR, Cattelan AM, Soriano Viladomiu A, et al. Effect of remdesivir vs standard care on clinical status at 11 days in patients with moderate COVID-19: A randomized clinical trial. JAMA. 2020;324(11):1048. DOI:10.1001/ jama.2020.16349

13. Zhou Q, Chen V, Shannon CP, Wei X-S, Xiang $X$, Wang $X$, et al. Interferon-a2b treatment for COVID-19. Front. Immunol. 2020;11:1061. DOl:10.3389/fimmu.2020.01061

14. Krolewiecki A, Lifschitz A, Moragas M, Travacio $M$, Valentini $R$, Alonso DF, et al. Antiviral effect of high-dose ivermectin in adults with COVID-19: A pilot randomised, controlled, open label, multicentre trial. SSRN Electron. J. 2020. DOI: 10.2139/ ssrn.3714649

15. Gorial Fl, Mashhadani S, Sayaly HM, Dakhil BD, AlMashhadani MM, Aljabory AM, et al. Effectiveness of ivermectin as add-on therapy in COVID-19 Management (Pilot Trial). Infectious Diseases (except HIV/AIDS); 2020

16. The RECOVERY Collaborative Group. Dexamethasone in hospitalized patients with Covid-19. N. Engl. J. Med. 2021;384(8):693-704. DOl: 10.1056/ NEJMoa2021436

17. Ye M, Fu D, Ren Y, Wang F, Wang D, Zhang $F$, et al. Treatment with convalescent plasma for COVID-19 patients in Wuhan, China. J. Med. Virol. 2020;92(10):1890-1901. DOI: 10.1002/jmv.25882

18. Casadevall A, Pirofski L. The convalescent sera option for containing COVID-19. J. Clin. Invest. 2020;130(4):1545-1548. DOI: 10.1172/ JCl138003
19. Abolghasemi H, Eshghi P, Cheraghali AM, Imani Fooladi AA, Bolouki Moghaddam F, Imanizadeh S, et al. Clinical efficacy of convalescent plasma for treatment of COVID-19 infections: Results of a multicenter clinical study. Transfus. Apher. Sci. 2020;59(5):102875. DOI: 10.1016/j. transci.2020.102875

20. Agarwal A, Mukherjee A, Kumar G, Chatterjee P, Bhatnagar T, Malhotra P. Convalescent plasma in the management of moderate covid-19 in adults in India: open label phase II multicentre randomised controlled trial (PLACID Trial). BMJ. 2020 Oct 22:m3939. DOI: 10.1136/bmj.m3939

21. Rossetti CA, Real JP, Palma SD. Utilización de altas dosis de ácido ascórbico en el tratamiento del SARS Covid-19: Soporte Científico y Clínico para su aplicación en terapéutica. Ars Pharm. 2020;61 (2):145-8. DOI: 10.30827/ars.v61i2.15164

22. Luo P, Liu Y, Qiu L, Liu X, Liu D, Li J. Tocilizumab treatment in COVID-19: A single center experience. J. Med. Virol. 2020;92(7):814-818. DOI: 10.1002/jmv.25801

23. RECOVERY Collaborative Group, Horby PW, Pessoa-Amorim G, Peto L, Brightling CE, Sarkar R, et al. Tocilizumab in patients admitted to hospital with COVID-19 (RECOVERY): preliminary results of a randomised, controlled, open-label, platform trial. Infectious Diseases (except HIV/AIDS); 2021. DOI: 10.1101/2021.02.11.21249258

24. Gautret $P$, Lagier J-C, Parola P, Hoang VT, Meddeb L, Mailhe M, et al. Hydroxychloroquine and azithromycin as a treatment of COVID-19: results of an open-label non-randomized clinical trial. Int. J. Antimicrob. Agents. 2020;56(1):105949. DOI: 10.1016/j.ijantimicag.2020.105949

25. Tang W, Cao Z, Han M, Wang Z, Chen J, Sun $W$, et al. Hydroxychloroquine in patients mainly with mild to moderate COVID-19: an open-label, randomized, controlled trial. Public and Global Health; 2020. DOI: 10.1101/2020.04.10.20060558 